Ce numéro du Forestry Chronicle porte sur l'histoire de notre journal. Depuis 75 ans, le Chronicle a diffusé les nouvelles et le point de vue des praticiens de la foresterie. Tel que Jim Cayford l'a noté dans sa révision historique, le journal a changé de taille, de format et de contenu au cours de ses 75 ans, mais le mandat principal de diffusion de l'information aux membres de l'Institut est demeuré en place. Nous encourageons les membres à soumettre des articles professionnels et scientifiques au journal, et à transmettre de l'information sur ce qui se passe dans leur région.

Il y a quelques semaines, la Fédération canadienne des associations de forestiers professionnels (FCAFP) a tenu une réunion d'une semaine à Edmonton, en Alberta. Pendant trois jours, il y a eu des réunions avec les représentants des écoles de foresterie qui offrent des programmes de baccalauréat en foresterie et le Bureau canadien d'agrément en foresterie pour discuter des aptitudes requises chez un forestier. Lorne Riley, le directeur exécutif du Bureau, a informé les participants sur les programmes d'agrément en foresterie. Ted Needham et Pierre Zundel de l'Université du NouveauBrunswick ont donné de l'information sur leurs travaux de recherche DACCUM. Ces deux présentations ont permis d'établir les prémisses nécessaires aux discussions. Richard Rothwell a élaboré le canevas des discussions des trois jours qui ont suivi. Les résultats de cet atelier sur les Aptitudes des forestiers seront rédigés et publiés dans un article d'un prochain numéro du Forestry Chronicle prévu pour le début de 2001.

Un atelier de deux jours a suivi ces trois premiers jours, et a porté sur la Mobilité de la main d'œuvre. Le ministère du Développement des ressources humaines du Canada a apporté l'aide financière aux organismes membres de la FCAFP (Association of British Columbia Professional Foresters, Alberta Registered Professional Foresters Association, Ontario Professional Foresters Association, Ordre des ingénieurs forestiers du Québec, Association des forestiers professionnels inscrits du Nouveau-Brunswick, Nova Scotia Professional Foresters Association, CIF/IFC Newfoundland and Labrador Registered Professional Foresters Association ainsi que l'Institut forestier du Canada, afin de leur permettre de se rencontrer et de discuter des enjeux qui entourent la mobilité de la main d'œuvre et des éléments nécessaires pour répondre à l'Article 7 de l'Accord sur le commerce interne. Dianne Blum et Stuart Clark, représentants des ministères responsables de la

\section{Executive Director}

Northern Forestry Centre and their hospitality is appreciated. The CFPFA members will meet again in November 2000 to further their discussions in this area. A "Mutual recognition agreement" between the regulatory bodies will be developed, and signed by the member agencies by July 1,2001 . The CIF/IFC provides secretariat services to the CFPFA, and also represents those provinces without RPF associations. The Chair of the CFPFA rotates annually, and this year, CIF/IFC holds the position of Chair, and ABCPF is the vice-Chair. section sur la mobilité de la main d'œuvre étaient également sur place pour représenter l'Alberta et la Colombie-Britannique.

Claudia Newman a agit en tant que facilitateur au cours de cet atelier, et un rapport final reposant sur les discussions des membres sera rédigé. Un tableau a été élaboré à partir des éléments requis par chaque association. L'ARPFA a organisé ces ateliers au Centre de foresterie du Nord et leur hospitalité a été grandement appréciée. Les membres de la FCAFP se réuniront à nouveau en novembre 2000 pour poursuivre leurs discussions dans ce domaine. Un « Accord de reconnaissance mutuelle » entre les organismes régulateurs sera élaboré et signé par les organismes membres le 1 juillet 2001. L'Institut fournit les services de secrétariat à la FCAFP, et représente également les provinces sans association de forestiers professionnels. La présidence de la FCAFP change chaque année, et en 2000, l'Institut détient la présidence, et l'ABCPF siège à la vice-présidence.

\section{Come visit CIF/IFC at http://www.cif-ifc.org}

read about CIF/IFC activities \& Section News

find Working Group contacts

read CIF/IFC Position Papers

learn about Continuing Education Opportunities

a explore Job Opportunities

become a member

view links to other sites

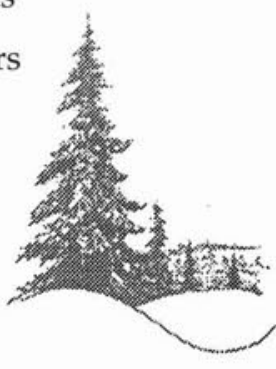

\section{Are You Moving?}

Please help us to keep our records up to date. Let us know when your address changes. There are four ways to tell us:

1. Email us at cif@cif-ifc.org

2. Fax us at (613) 234-6181

3. Telephone us at (613) 234-2242

4. Mail us at 151 Slater Street, Suite 606, Ottawa, ON KIP $5 \mathrm{H} 3$. 